\title{
Are LGRBs biased tracers of star formation? Clues from the host galaxies of the Swift/BAT6 complete sample of LGRBs
}

\author{
Susanna D. Vergani ${ }^{1,2}$ and collaborators \\ ${ }^{1}$ GEPI, Observatoire de Paris, PSL Research University, CNRS, \\ Univ Paris Diderot, Sorbonne Paris Cité, \\ Place Jules Janssen, 92195 Meudon, France \\ email: susanna.vergani@obspm.fr \\ ${ }^{2}$ INAF, Osservatorio Astronomico di Brera, via E. Bianchi 46, 23807 Merate, Italy
}

\begin{abstract}
Long gamma-ray bursts (LGRBs) are associated to the deaths of massive stars and could thus be used as a potentially powerful tool to trace cosmic star formation. However the conditions needed to produce a LGRBs may introduce a bias in the LGRB rate versus star formation rate (SFR) relation (called LGRB efficiency hereafter).

We have undertaken a study of the properties of the host galaxies of the BAT6 complete sample of LGRB to improve our knowledge on the LGRB efficiency, its redshift evolution, and the factor affecting it. This is the base to properly use LGRBs as SFR tracers.

We show that at $z<1$ LGRBs are not direct SFR tracers because they tend to avoid highmetallicity galaxies. The use of the BAT6 complete sample keeps this result from being affected by possible biases that could have influenced past results based on incomplete samples. The preference for low (but not extremely low) metallicities can be a consequence of the particular conditions needed for the progenitor star to produce a GRB.
\end{abstract}

Keywords. gamma rays: bursts, galaxies: fundamental parameters

\section{Introduction}

We have undertaken a study of the properties of the host galaxies of the BAT6 complete sample of LGRBs (Salvaterra et al. 2012). The sample consists of 58 LGRBs, and it is complete in redshift at $97 \%$. This sample offers the opportunity to study the population of LGRBs in a statistically unbiased way. In particular, as the brightness of the gamma-ray prompt emission is the only burst-depending parameter for selecting the LGRB sample, the BAT6 allows the selection of a complete sample of LGRB host galaxies independently of their fluxes or colours. Our project is to study the properties of the host galaxies of the LGRBs of the BAT6 with the aim of investigating the relation between LGRB and star formation.

We present here the results obtained with the study of the stellar masses of the BAT6 LGRB host galaxies at $z<1$ and the preliminary results on the SFR and metallicity (Z) obtained through the spectroscopy of the same galaxies.

\section{Results}

We show in Fig. 1 the results of our photometric and spectroscopic campaigns, demonstrating that LGRB are not direct tracers of the SFR and that they tend to avoid high-Z galaxies. 

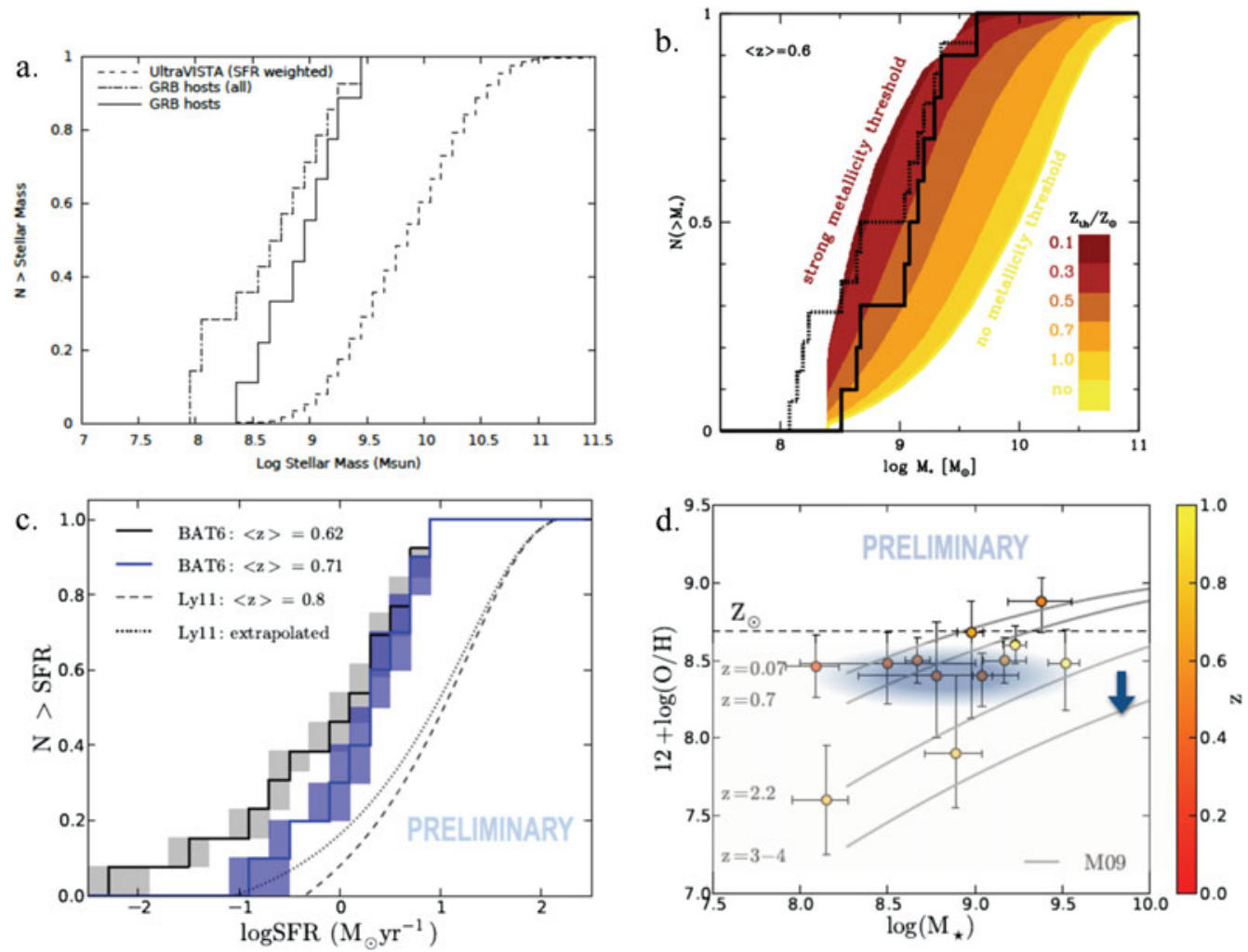

Figure 1. a. Comparison between the stellar mass distribution of the BAT6 $z<1$ LGRB host galaxies and those of field star-forming galaxies weighted by the SFR, showing that LGRBs are not direct SFR tracers (see Vergani et al. 2015 for more details). b. Comparison of the the stellar mass distribution of the BAT $6 z<1$ LGRB host galaxies with simulations of LGRB host galaxies obtained imposing different metallicity thresholds for the LGRB progenitor. The distribution is consistent with a threshold around $\mathrm{Z}=0.5 \mathrm{Z}_{\odot}$. The case of no threshold or extremely low thresholds are excluded (see Vergani et al. 2015 for more details). c. Comparison of the SFR distribution of the BAT6 $z<1$ LGRB host galaxies and that of field star-forming galaxies (SFR weighted), showing that LGRBs are not direct SFR tracers (Japelj et al. in preparation). d. M-Z relation of the BAT6 $z<1$ LGRB host galaxies, confirming that LGRBs tend to avoid high $\mathrm{Z}$ galaxies and showing that they follow the mass-Z relation (Japelj et al. in preparation).

\section{Conclusions}

LGRBs at $z<1$ seem not to be direct tracers of star formation. The LGRB efficiency is affected by the preference of LGRBs to happen in low-metallicity galaxies (but in general consistent with the mass- $Z$ relation). It is still not clear if metallicity is the only factor at play. Our results can also have some impact on LGRB progenitor model studies and could discard models needing extremely low metallicities for the LGRB production.

\section{References}

Salvaterra, R., Campana, S., \& Vergani, S. D., et al. 2012, ApJ, 749, 68

Vergani, S. D., Salvaterra, R., \& Japelj, J., et al. 2015, A\&BA, 581, 102 